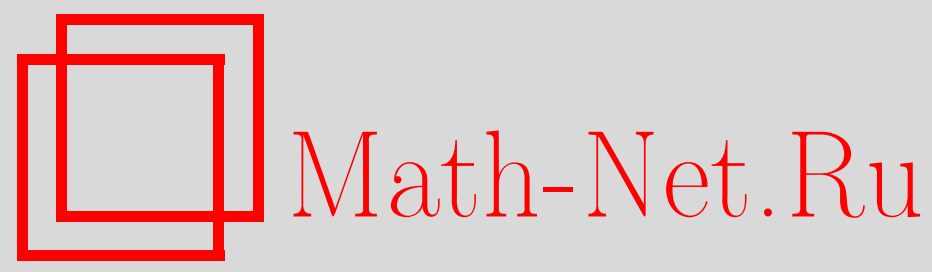

А. В. Шкляев, Большие уклонения ветвящихся процессов в случайной среде с произвольным начальным числом частиц, Дискрет. матем., 2012, том 24, выпуск 4, 114-130

DOI: https://doi.org/10.4213/dm1215

Использование Общероссийского математического портала Math-Net.Ru подразумевает, что вы прочитали и согласны с пользовательским соглашением http://www . mathnet.ru/rus/agreement

Параметры загрузки:

IP : 54.210 .77 .194

26 апреля 2023 г., $12: 33: 46$ 


\title{
Большие уклонения ветвящихся процессов в случайной среде с произвольным начальным числом частищ
}

\author{
() 2012 г. А. В. Шкляев
}

Нами рассматривается ветвящийся процесс $Z_{n}$ с дробно-линейной производящей функцией распределения числа непосредственных потомков в случайной среде $\eta=\left(\eta_{1}, \ldots, \eta_{n} \ldots\right)$, представляющей собой последовательность независимых одинаково распределенных величин. Известно, что при определенных условиях имеет место эквивалентность

$$
\mathbf{P}\left(\ln Z_{n} \geqslant \theta n \mid Z_{0}=1\right) \sim I(\theta) \mathbf{P}\left(S_{n} \geqslant \theta n\right)
$$

при $n \rightarrow \infty, I(\theta)>0$, где $S_{n}$ есть сопровождающее случайное блуждание. В этих условиях в работе получена асимптотика вероятностей больших уклонений $\mathbf{P}\left(\ln Z_{n} \geqslant \ln m+\theta n \mid Z_{0}=m\right)$ при $n \rightarrow \infty$ и $m$ конечном или стремящемся к бесконечности.

\section{1. Введение}

Пусть $\eta=\left(\eta_{0}, \ldots, \eta_{n}, \ldots\right)-$ последовательность независимых одинаково распределенных случайных величин и $f_{y}(s), y \in \mathbf{R},-$ семейство вероятностных производящих функций. Ветвящийся процесс $Z_{n}$ в случайной среде $\eta$ представляет собой марковскую цепь с условной производящей фунцией переходных вероятностей, равной (см., например, $[1,2])$

$$
\mathbf{E}\left(s^{Z_{n+1}} \mid Z_{n}, \eta\right)=f_{\eta_{n}}(s)^{Z_{n}} \text { п.н. }
$$

В настоящей работе рассматривается случай дробно-линейных производящих функций. Легко проверить, что

$$
f_{y}(s)=1-\left(\frac{b(y)}{2 a(y)^{2}}+\frac{1}{(1-s) a(y)}\right)^{-1}, \quad a(y)=f_{y}^{\prime}(1), \quad b(y)=f_{y}^{\prime \prime}(1) .
$$

Положим

$$
\begin{aligned}
& X_{i}=\ln a\left(\eta_{i-1}\right), \quad \zeta_{i}=\ln \frac{b\left(\eta_{i}\right)}{2 a^{2}\left(\eta_{i}\right)}, \quad i>0, \\
& S_{n}=\sum_{i=1}^{n} X_{i}, \quad n>0, \quad S_{0}=0, \\
& U_{n}=e^{-S_{n}}, \quad V_{n}=\sum_{i=0}^{n-1} e^{-S_{i}+\zeta_{i}},
\end{aligned}
$$


и пусть $X$ - общее обозначение для $X_{i}$.

Случайное блуждание $S_{n}$ называют сопровождающим для ветвящегося процесса в случайной среде $Z_{n}$. Будем предполагать, что выполняется правостороннее условие Крамера:

$$
\mathbf{E} e^{h X}<\infty, \quad h \in\left(0, h^{+}\right),
$$

случайная величина $X$ невырождена, нерешетчата и имеет конечное математическое ожидание. От величин $\zeta_{i}$ потребуем выполнения правостороннего условия Крамера при всех $h>0$.

Условную производящую функцию случайной величины $Z_{n}$ запишем в виде [3]

$$
\mathbf{E}\left(s^{Z_{n}} \mid \eta, Z_{0}=1\right)=1-\left(\frac{U_{n}}{1-s}+V_{n}\right)^{-1} .
$$

Легко проверить, что

$$
\begin{array}{rlrl}
\mathbf{P}\left(Z_{n}>\right. & \left.k \mid Z_{0}=1, \eta\right)=\left(V_{n}+U_{n}\right)^{-1}\left(1+V_{n}^{-1} U_{n}\right)^{-k}, & & k \geqslant 0, \\
& \mathbf{P}\left(Z_{n}=k \mid \eta\right)=U_{n} V_{n}^{k-1}\left(V_{n}+U_{n}\right)^{-k-1}, & & k>0, \\
& \mathbf{P}\left(Z_{n}=0 \mid \eta\right)=1-\left(V_{n}+U_{n}\right)^{-1} . &
\end{array}
$$

Мы связываем вероятности больших уклонений $\mathbf{P}\left(\ln Z_{n} \geqslant \ln m+\theta n \mid Z_{0}=m\right)$ с вероятностями $\mathbf{P}\left(S_{n} \geqslant \theta n\right)$ при $\theta \in\left(\mu, m^{+}\right)$в случае $\mu=\mathbf{E} X \geqslant 0$ и при $\theta \in\left(\theta^{*}, m^{+}\right)$в случае $\mu<0\left(\theta^{*}, m^{+}\right.$определены в разделе 2$)$. Конечное $m$ рассматривается в разделе 3 , $m$, растущее вместе с $n,-$ в разделе 4 .

Случай $m=1$ хорошо изучен для геометрического распределения числа непосредственных потомков одной частицы. В $[1,2]$ получена точная асимптотика вероятностей больших уклонений, грубая асимптотика получена в [7, 8] для широкого класса распределений. Изучение вероятностей $\mathbf{P}\left(\ln Z_{n} \geqslant \ln m+\theta n \mid Z_{0}=m\right)$ при больших $m$ оказывается важным при асимптотическом анализе траектории процесса $Z_{n}$ при условии большого уклонения $\ln Z_{n} \geqslant \theta n$.

В случае конечного $m$ вывод асимптотики вероятностей больших уклонений при $b(y) /\left(2 a^{2}(y)\right)=1$ несущественно отличается от общего, и ради краткости мы ограничиваемся этим частным случаем. Для $m$, растущего вместе с $n$, это ограничение остается в леммах 1,4 , тогда как леммы 2 и 3 доказываются в случае произвольной дробно-линейной производящей функции ввиду необходимости дополнительных оценок.

\section{2. Обозначения и предварительные сведения}

Пусть

$$
S_{n}=\sum_{i=1}^{n} X_{i}
$$

- случайное блуждание, $-\infty<\mu=\mathbf{E} X<\infty$, случайная величина $X$ нерешетчатая,

$$
R(h)=\mathbf{E} e^{h X}<\infty, \quad h \in\left(0, h^{+}\right) .
$$

Положим

$$
m(h)=(\ln R(h))^{\prime}, \quad \sigma^{2}(h)=m^{\prime}(h)>0, \quad m^{+}=\lim _{h \rightarrow h^{+}} m(h) .
$$


Так как $\sigma^{2}(h)>0$, функция $m(h)$ монотонно возрастает при $h \in\left(0, h^{+}\right), m(0)=\mu$, следовательно, при любом $\theta \in\left(\mu, m^{+}\right)$найдется такое $h_{\theta} \in\left(0, h^{+}\right)$, что $m\left(h_{\theta}\right)=\theta$.

Функцией уклонений называется функция

$$
\Lambda(\theta)=\theta h_{\theta}-\ln R\left(h_{\theta}\right)
$$

Пусть $X_{i}^{(\theta)}-$ независимые случайные величины, одинаково распределенные с функцией распределения

$$
F^{(\theta)}(x)=\mathbf{P}\left(X^{(\theta)} \leqslant x\right)=R\left(h_{\theta}\right)^{-1} \int_{-\infty}^{x} e^{h_{\theta} y} d F(y) .
$$

Функция распределения $F^{(\theta)}$ называется преобразованием Крамера функции распределения $F$. Блуждание

$$
S_{n}^{(\theta)}=\sum_{i=1}^{n} X_{i}^{(\theta)}
$$

называется сопряженным к $S_{n}$. Нам потребуются следующие результаты из теории больших уклонений $[4,5]$ :

$$
\begin{aligned}
\mathbf{P}\left(S_{n} \geqslant \theta n\right) & \sim \frac{1}{\sqrt{2 \pi n} h_{\theta} \sigma\left(h_{\theta}\right)} e^{-\Lambda(\theta) n}, & & n \rightarrow \infty, \\
\mathbf{P}\left(\max _{i \leqslant n} S_{i} \geqslant \theta n\right) & \sim D(\theta) e^{-\Lambda(\theta) n} n^{-1 / 2}, & & n \rightarrow \infty,
\end{aligned}
$$

причем соотношение (4) выполняется равномерно по $\theta \in\left[\theta_{1}, \theta_{2}\right] \subset\left(\mu, m^{+}\right)$, а соотношение (5) - равномерно по

$$
\theta \in\left[\theta_{1}, \theta_{2}\right] \subset \begin{cases}\left(\mu, m^{+}\right), & \text {в случае, когда } \mathbf{E} X \geqslant 0, \\ \left(\theta^{*}, m^{+}\right) & \text {в случае, когда } \mathbf{E} X<0 .\end{cases}
$$

где $\theta^{*}>0$ определяется из соотношения

$$
R\left(h_{\theta *}\right)=1 .
$$

Существенную роль в дальнейших построениях играет следующее утверждение для ветвящихся процессов в случайной среде с геометрическим распределением числа непосредственных потомков одной частицы [1]:

$$
\mathbf{P}\left(Z_{n} \geqslant e^{\theta n} \mid Z_{0}=1\right) \sim \mathbf{P}\left(S_{n} \geqslant \theta n\right) I(\theta), \quad n \rightarrow \infty,
$$

равномерно по $\theta$, указанным в (6), где Г(·) есть гамма-функция,

$$
I(\theta)=h_{\theta} \Gamma\left(h_{\theta}\right) \int_{1}^{\infty} v^{h_{\theta}-1} \mathbf{P}\left(\sum_{j=0}^{\infty} e^{-S_{j}^{(\theta)}} \in d x\right) .
$$

Замечание 1. Заметим, что в [1], в определении $I(\theta) \equiv I_{1}(\theta)$, пропущен множитель $h_{\theta}$. 


\section{3. Случай конечного $m$}

Рассмотрим ветвящийся процесс в случайной среде $Z_{n}$, шаги $X_{i}$ сопровождающего блуждания $S_{n}$ которого нерешетчаты, удовлетворяют правостороннему условию Крамера и имеют конечное математическое ожидание. Потребуем также, чтобы $\mathbf{E} e^{h \zeta_{i}}<\infty$ при всех $h>0$. Для ветвящегося процесса в случайной среде с геометрическим распределением числа частиц положим

$$
V^{(\theta)}=\sum_{i=0}^{\infty} \exp \left(-S_{i}^{(\theta)}\right), \quad S_{0}^{(\theta)}=0
$$

а в случае дробно-линейной производящей функции положим

$$
V^{(\theta)}=\sum_{j=0}^{\infty} \frac{b\left(\eta_{j}^{(\theta)}\right)}{2 a^{2}\left(\eta_{j}^{(\theta)}\right)} \exp \left\{-\sum_{i=1}^{j-1} \ln a\left(\eta_{i}^{(\theta)}\right)\right\}
$$

где $\eta_{i}^{(\theta)}$ есть последовательность независимых случайных величин, одинаково распределенных по закону

$$
\mathbf{P}\left(\eta_{i}^{(\theta)} \in d x\right)=\mathbf{P}\left(\eta_{i} \in d x\right) e^{h_{\theta} \ln a(x)} R\left(h_{\theta}\right)^{-1} .
$$

Теорема 1. При $n \rightarrow \infty$ и произвольном натуральном $m$, соотношение

$$
\mathbf{P}\left(\ln Z_{n} \geqslant \theta n+\ln m \mid Z_{0}=m\right) \sim I_{m}(\theta) \mathbf{P}\left(S_{n} \geqslant \theta n\right),
$$

где

$$
I_{m}(\theta)=h_{\theta} m^{-h_{\theta}} \sum_{k=0}^{m-1} \frac{\Gamma\left(h_{\theta}+i+1\right)}{i !} \sum_{i=0}^{m-k-1}\left(\begin{array}{l}
m \\
k
\end{array}\right) \mathbf{E}\left(\left(V^{(\theta)}\right)^{h_{\theta}-m+k}\left(1-\left(V^{(\theta)}\right)^{-1}\right)^{k}\right),
$$

выполнено равномерно по $\theta$, указанным в (6).

Доказательство. Доказательство будет проведено для ветвящихся процессов в случайной среде с геометрическим распределением числа потомков одной частицы; об изменениях, необходимых в дробно-линейном случае, будет сказано в замечании 2.

Представим ветвящийся процесс в случайной среде $Z_{n}$ с $m$ начальными частицами в виде суммы $m$ процессов $Z_{n}^{(i)}$, условно независимых при условии среды $\eta$, стартующих с одной частицы. Тогда

$$
\mathbf{P}\left(Z_{n} \geqslant e^{\theta n} m \mid Z_{0}=m\right)=\mathbf{P}\left(Z_{n}^{(1)}+\ldots+Z_{n}^{(m)} \geqslant e^{\theta n} m\right) .
$$

В силу лемм 6,11 и 12 в [1], для заданного $m$, произвольного $\varepsilon>0$ и достаточно большого $N$ найдется такое $M$, что при всех $n>N$ имеет место неравенство

$$
\mathbf{E}\left(\mathbf{P}\left(Z_{n}^{(i)} \geqslant e^{\theta n} \mid \eta\right) I_{S_{n} \leqslant \theta n-M}\right) \leqslant \frac{\varepsilon}{4 m} \mathbf{P}\left(S_{n} \geqslant \theta n\right),
$$

где $I_{A}$ обозначает индикатор события $A$. Следовательно, при всех $n>N$

$$
\begin{aligned}
\mathbf{E}\left(\mathbf{P}\left(Z_{n} \geqslant e^{\theta n} m \mid Z_{0}=m, \eta\right) I_{S_{n}<\theta n-M}\right) & =\mathbf{P}\left(Z_{n}^{(1)}+\ldots+Z_{n}^{(m)} \geqslant e^{\theta n} m, S_{n}<\theta n-M\right) \\
& \leqslant \sum_{i=1}^{n} \mathbf{P}\left(Z_{n}^{(i)} \geqslant e^{\theta n}, S_{n} \leqslant \theta n-M\right) \\
& \leqslant \frac{\varepsilon}{4} \mathbf{P}\left(S_{n} \geqslant \theta n\right) .
\end{aligned}
$$


Покажем, что при условии $\left\{S_{n}-\theta n \geqslant-M\right\}$ величины $V_{n}$ сходятся при $n \rightarrow \infty$ по распределению к

$$
V^{(\theta)}=\sum_{i=0}^{\infty} e^{-S_{i}^{(\theta)}}
$$

Проводя преобразование Крамера и применяя лемму 5 из [1], получаем, что

$$
\begin{aligned}
P\left(V_{n} \geqslant y, S_{n} \geqslant \theta n-M\right) & =R\left(h_{\theta}\right)^{-n} \int_{-M}^{\infty} e^{-h_{\theta} x-h_{\theta} \theta n} \mathbf{P}\left(V_{n}^{(\theta)} \geqslant y, S_{n}^{(\theta)}-\theta n \in d x\right) \\
& \sim \frac{e^{-\Lambda(\theta) n}}{\sigma\left(h_{\theta}\right) \sqrt{2 \pi n}} \mathbf{P}\left(V^{(\theta)} \geqslant y\right) \int_{-M}^{\infty} e^{-h_{\theta} x} d x,
\end{aligned}
$$

где $y$ есть точка непрерывности функции распределения случайной величины $V^{(\theta)}$. Следовательно, для любых $M, \varepsilon$ и всех достаточно больших $C$ выполняется неравенство

$$
\mathbf{P}\left(V_{n} \geqslant C, S_{n} \geqslant \theta n-M\right)<\frac{\varepsilon}{4} \mathbf{P}\left(S_{n} \geqslant \theta n\right) .
$$

Перейдем к оценке величины $\mathbf{E}\left(\mathbf{P}\left(Z_{n} \geqslant e^{\theta n} m \mid Z_{0}=m, \eta\right) I_{S_{n} \geqslant \theta n-M}\right)$. Положим

$$
\begin{aligned}
I_{m, k} & =\left\{i_{1}, \ldots, i_{k}: 0<i_{1}<i_{2}<\ldots<i_{k}<m\right\}, \\
L_{m, l, i_{1}, \ldots, i_{k}} & =\left\{l_{1}, \ldots, l_{m}: l_{i_{1}}=0, \ldots, l_{i_{k}}=0, l_{1}+\ldots+l_{m}=l\right\},
\end{aligned}
$$

и $u=\left\lceil e^{\theta n} m\right\rceil$, где $\lceil x\rceil$ обозначает наименьшее целое число, не меньшее, чем $x$. Используя равенства (2) и (3), получаем, что

$$
\begin{aligned}
\mathbf{P}\left(Z_{n}^{(1)}+\right. & \left.\ldots+Z_{n}^{(m)} \geqslant e^{\theta n} m \mid \eta\right) \\
& =\sum_{l=u}^{\infty} \sum_{l_{1}+\ldots+l_{m}=l} \mathbf{P}\left(Z_{n}^{(1)}=l_{1}\right) \ldots \mathbf{P}\left(Z_{n}^{(m)}=l_{m}\right) \\
& =\sum_{l=u}^{\infty} \sum_{k=0}^{m-1} \sum_{I_{m, k}} \sum_{L_{m, i_{1}, \ldots, i_{k}}}\left(1-\frac{1}{V_{n}}\right)^{k} \prod_{i: l_{i}>0} \frac{U_{n} V_{n}^{l_{i}-1}}{\left(V_{n}+U_{n}\right)^{l_{i}+1}} \\
& =\sum_{l=u}^{\infty} \sum_{k=0}^{m-1}\left(\begin{array}{l}
m \\
k
\end{array}\right)\left(\begin{array}{c}
l-1 \\
m-k-1
\end{array}\right)\left(1-\frac{1}{V_{n}}\right)^{k}\left(\frac{U_{n}}{V_{n}\left(V_{n}+U_{n}\right)}\right)^{m-k}\left(\frac{V_{n}}{V_{n}+U_{n}}\right)^{l} \\
& =\sum_{k=0}^{m-1}\left(\begin{array}{l}
m \\
k
\end{array}\right)\left(1-\frac{1}{V_{n}}\right)^{k}\left(\frac{U_{n}}{V_{n}\left(V_{n}+U_{n}\right)}\right)^{m-k} \sum_{l=u}^{\infty}\left(\begin{array}{c}
l-1 \\
m-k-1
\end{array}\right)\left(\frac{V_{n}}{V_{n}+U_{n}}\right)^{l} .
\end{aligned}
$$

Полагая для краткости

$$
a=\frac{V_{n}}{V_{n}+U_{n}},
$$


преобразуем сумму

$$
\begin{aligned}
\sum_{l=u}^{\infty} a^{l}\left(\begin{array}{c}
l-1 \\
m-k-1
\end{array}\right) & =\frac{a^{m-k}}{(m-k-1) !} \sum_{l=u}^{\infty}(l-1) \cdots(l-m+k+1) a^{l-m+k} \\
& =\frac{a^{m-k}}{(m-k-1) !} \sum_{l=u}^{\infty} \frac{d^{m-k-1} a^{l-1}}{d a^{m-k-1}} \\
& =\frac{a^{m-k}}{(m-k-1) !}\left(\sum_{l=u}^{\infty} a^{l-1}\right)^{(m-k-1)} \frac{a^{m-k}}{(m-k-1) !}\left(\frac{a^{u-1}}{1-a}\right)^{m-k-1} \\
& =\frac{a^{m-l}}{(m-k-1) !} \sum_{i=0}^{m-k-1}\left(\begin{array}{c}
m-k-1 \\
i
\end{array}\right)\left(a^{u-1}\right)^{(i)}\left(\frac{1}{1-a}\right)^{m-k-1-i} \\
& =\frac{a^{m-k}}{(m-k-1) !} \sum_{i=0}^{m-k-1}\left(\begin{array}{c}
m-k-1 \\
(m-k
\end{array}\right)(u-1) \cdots(u-i-1) \\
& =a^{u-1} \sum_{i=0}^{m-k-1}\left(\begin{array}{l}
u-1 \\
a^{u-1-i}(m-k-i-1) ! \\
i
\end{array}\right.
\end{aligned}
$$

Подставляя (10) в (9), получаем, что

$$
\begin{aligned}
\mathbf{P}\left(Z_{n}^{(1)}+\ldots+Z_{n}^{(m)} \geqslant e^{\theta n} m\right. & \mid \eta)=\left(\frac{V_{n}}{V_{n}+U_{n}}\right)^{u-1} \sum_{k=0}^{m-1}\left(\begin{array}{c}
m \\
k
\end{array}\right)\left(1-\frac{1}{V_{n}}\right)^{k} \\
& \times\left(\frac{U_{n}}{V_{n}\left(V_{n}+U_{n}\right)}\right)^{m-k} \sum_{i=0}^{m-k-1}\left(\begin{array}{c}
u-1 \\
i
\end{array}\right)\left(\frac{U_{n}}{V_{n}}\right)^{-m+k+i} .
\end{aligned}
$$

Пользуясь тем, что

$$
\left(\begin{array}{c}
u-1 \\
i
\end{array}\right)=(1+o(1)) \frac{u^{i}}{i !}, \quad u \rightarrow \infty, \quad i<u,
$$

получаем, что при условии события $\left\{S_{n} \geqslant \theta n-M, V_{n}<C\right\}$ при $n \rightarrow \infty$ выражение (11) эквивалентно, равномерно по $\theta$ из (6), следующему выражению:

$$
\begin{aligned}
& \left(1-\frac{U_{n}}{V_{n}+U_{n}}\right)^{u-1} \sum_{k=0}^{m-1}\left(\begin{array}{c}
m \\
k
\end{array}\right)\left(1-\frac{1}{V_{n}}\right)^{k}\left(\frac{1}{V_{n}+U_{n}}\right)^{m-k} \sum_{i=0}^{m-k-1} \frac{\left(m e^{\theta n-S_{n}}\right)^{i}}{i !} \frac{1}{V_{n}^{i}} \\
& =e^{-m\left(V_{n}+U_{n}\right)^{-1} e^{-\Delta_{n}}} \sum_{k=0}^{m-1}\left(\begin{array}{l}
m \\
k
\end{array}\right)\left(1-\frac{1}{V_{n}}\right)^{k}\left(\frac{1}{V_{n}+U_{n}}\right)^{m-k} \sum_{i=0}^{m-k-1} \frac{\left(m e^{-\Delta_{n}}\right)^{i}}{i !} \frac{1}{V_{n}^{i}},
\end{aligned}
$$

где полагаем

$$
\Delta_{n}=S_{n}-\theta n .
$$


Математическое ожидание выражения (11), суженное на событие $\left\{S_{n} \geqslant \theta n-M, V_{n}<C\right\}$, эквивалентно, с учетом (12), следуюшему выражению:

$$
\begin{gathered}
\mathbf{E}\left(e^{-m e^{-\Delta_{n}}\left(V_{n}+U_{n}\right)^{-1}} \sum_{k=0}^{m-1}\left(\begin{array}{c}
m \\
k
\end{array}\right)\left(1-\frac{1}{V_{n}}\right)^{k}\left(\frac{1}{V_{n}+U_{n}}\right)^{m-k}\right. \\
\left.\quad \times \sum_{i=0}^{m-k-1} \frac{\left(m e^{-\Delta_{n}}\right)^{i}}{i !} \frac{1}{V_{n}^{i}} I_{S_{n}-\theta n>M, V_{n}<C}\right) \\
=e^{-\Lambda(\theta) n} \int_{-M}^{M} \int_{1}^{C} e^{-h_{\theta} x} e^{-m e^{-x} y} \sum_{k=0}^{m-1}\left(\begin{array}{l}
m \\
k
\end{array}\right)\left(1-y^{-1}\right)^{k} y^{-m+k} \\
\quad \times \sum_{i=0}^{m-k-1} \frac{m^{i} e^{-x i} y^{-i}}{i !} \mathbf{P}\left(S_{n}^{(\theta)}-\theta n \in d x, V_{n}^{(\theta)} \in d y\right) .
\end{gathered}
$$

Ввиду леммы 5 из [1], для любого $M>0$ на множестве $[-M, M] \times[1, \infty)$ имеет место слабая сходимость мер

$$
\sqrt{n} \mathbf{P}\left(S_{n}^{(\theta)}-\theta n \in d x, V_{n}^{(\theta)} \in d y\right) \Rightarrow \lambda(d x) \mathbf{P}\left(V^{(\theta)} \in d y\right)\left(\sqrt{2 \pi} \sigma\left(h_{\theta}\right)\right)^{-1}, \quad n \rightarrow \infty,
$$

где $\lambda$ есть мера Лебега на прямой. Подставим это соотношение в (13) и, пользуясь ограниченностью подынтегральной функции, получим следующее эквивалентное при $n \rightarrow \infty$ выражение для (13):

$$
\begin{aligned}
\frac{e^{-\Lambda(\theta) n}}{\sigma\left(h_{\theta}\right) \sqrt{2 \pi n}} \int_{-M}^{M} \int_{1}^{C} e^{-m e^{-x} y^{-1}} \sum_{k=0}^{m-1}\left(\begin{array}{l}
m \\
k
\end{array}\right)\left(1-y^{-1}\right)^{k} y^{-m+k} & \\
& \times \sum_{i=0}^{m-k-1} \frac{m^{i} e^{-x\left(h_{\theta}+i\right)} y^{-i}}{i !} \mathbf{P}\left(V^{(\theta)} \in d y\right) d x .
\end{aligned}
$$

Учитывая соотношения (7), (8), получаем, что

$$
\begin{aligned}
& \mid \mathbf{P}\left(Z_{n} \geqslant e^{\theta n} \mid Z_{0}=m\right) \\
&-\frac{e^{-\Lambda(\theta) n}}{\sigma\left(h_{\theta}\right) \sqrt{2 \pi n}} \int_{-M}^{M} \int_{1}^{C} e^{-m e^{-x} y^{-1}} \sum_{k=0}^{m-1}\left(\begin{array}{l}
m \\
k
\end{array}\right)\left(1-y^{-1}\right)^{k} y^{-m+k} \\
& \times \sum_{i=0}^{m-k-1} \frac{m^{i} e^{-x\left(h_{\theta}+i\right)} y^{-i}}{i !} \mathbf{P}\left(V^{(\theta)} \in d y\right) d x \mid \\
& \leqslant\left(\frac{\varepsilon}{2}+o_{n}\right) \mathbf{P}\left(S_{n} \geqslant \theta n\right),
\end{aligned}
$$

где $o_{n}$ стремится к нулю при $n \rightarrow \infty$ равномерно по $\theta \in\left[\theta_{1}, \theta_{2}\right]$. Устремляя в (15) $C$ и $M$ 
к бесконечности, а $\varepsilon$ к нулю, получаем, что

$$
\begin{aligned}
& \mathbf{P}\left(Z_{n}^{(1)}+\ldots+Z_{n}^{(m)} \geqslant e^{\theta n} m\right)=(1+o(1)) \mathbf{P}\left(S_{n} \geqslant \theta n\right) h_{\theta} \\
& \times \int_{1}^{\infty} \sum_{k=0}^{m-1}\left(1-y^{-1}\right)^{k} y^{-m+k}\left(\begin{array}{l}
m \\
k
\end{array}\right) \\
& \times \sum_{i=0}^{m-k-1} \frac{1}{i !} \int_{-\infty}^{\infty} e^{-m e^{-x} y^{-1}} m^{i} e^{-x\left(h_{\theta}+i\right)} y^{-i} d x \\
& \times \mathbf{P}\left(V^{(\theta)} \in d y\right) \\
& =(1+o(1)) \mathbf{P}\left(S_{n} \geqslant \theta n\right) h_{\theta} m^{-h_{\theta}} \\
& \times \int_{1}^{\infty} \sum_{k=0}^{m-1} y^{-m+k+h_{\theta}}\left(1-y^{-1}\right)^{k}\left(\begin{array}{l}
m \\
k
\end{array}\right) \\
& \times \sum_{i=0}^{m-k-1} \frac{1}{i !} \int_{-\infty}^{\infty} e^{-\left(m e^{-x} y^{-1}\right)}\left(m e^{-x} y^{-1}\right)^{h_{\theta}+i} m e^{-x} y^{-1} d x \\
& \times \mathbf{P}\left(V^{(\theta)} \in d y\right) \text {. }
\end{aligned}
$$

Для завершения доказательства теоремы остается произвести замену

$$
v=m e^{-x} y^{-1}
$$

во внутреннем интеграле.

Замечание 2. В приведенном доказательстве теоремы 1 фактически не используется явный вид $V_{n}, V^{(\theta)}$, а лишь результаты [1], относящиеся к случаю геометрического распределения непосредственного числа потомков одной частицы. Однако все они в условиях данной работы могут быть получены для ветвящегося процесса с случайной среде в дробно-линейном случае с помощью лемм 2, 3 раздела 4.

Замечание 3. Нами частично использована дипломная работа Д. Николаевой.

\section{4. Случай бесконечного $m$}

В предположениях, сформулированных в разделе 3 , справедливо следующее утверждение.

Теорема 2. Соотношение

$$
\mathbf{P}\left(\ln Z_{n} \geqslant \theta n+\ln m \mid Z_{0}=m\right) \sim \mathbf{P}\left(S_{n} \geqslant \theta n\right), \quad n \rightarrow \infty, \quad m \rightarrow \infty,
$$

выполняется равномерно по $\theta$, указанным в (6).

Доказательство. Разложим вероятность в (16) следующим образом:

$$
\begin{aligned}
& \mathbf{P}\left(Z_{n, m} \geqslant m e^{\theta n}\right)=\mathbf{E}\left(\mathbf{P}\left(Z_{n}^{(1)}+\ldots+Z_{n}^{(m)} \geqslant e^{\theta n} m \mid \eta\right)\right. \\
& \left.\times\left(I_{S_{n}<\theta n-M}+I_{\theta n-M \leqslant S_{n}<\theta n-\tilde{\varepsilon}}+I_{\theta n-\tilde{\varepsilon}<S_{n}<\theta n+\tilde{\varepsilon}}+I_{\theta n+\tilde{\varepsilon} \leqslant S_{n}<\theta n+M}+I_{S_{n} \geqslant \theta n+M}\right)\right),
\end{aligned}
$$

где $M, \tilde{\varepsilon}$ - некоторые параметры, а $Z_{n}^{(i)}$ - ветвящийся процесс, порожденный $i$-й начальной частицей. 
Лемма 1. Для любого $\varepsilon>0$ и всех достаточно больиих $M$ найдется такое $N$, что при $n>N$ и всех $m \geqslant 1$

$$
\mathbf{E}\left(\mathbf{P}\left(Z_{n}^{(1)}+\ldots+Z_{n}^{(m)} \geqslant e^{\theta n} m \mid \eta\right) I_{S_{n}<\theta n-M}\right)<\frac{\varepsilon}{5} \mathbf{P}\left(S_{n} \geqslant \theta n\right) .
$$

Доказательство. Полагая $M_{1}>2$, перепишем оцениваемое математическое ожидание в следующем виде:

$$
\mathbf{E}\left(\mathbf{P}\left(Z_{n}^{(1)}+\ldots+Z_{n}^{(m)} \geqslant e^{\theta n} m \mid \eta\right) I_{S_{n}<\theta n-M}\left(I_{U_{n} / V_{n} \geqslant M_{1} e^{-\theta n}}+I_{U_{n} / V_{n}<M_{1} e^{-\theta n}}\right)\right) .
$$

Для вероятности $\mathbf{P}\left(Z_{n}^{(1)}+\ldots+Z_{n}^{(m)} \geqslant e^{\theta n} m \mid \eta\right)$ при $S_{n}<\theta n-M$ и $U_{n} V_{n}^{-1} \geqslant M_{1} e^{-\theta n}$ используем эквивалентное ей при $n \rightarrow \infty$ выражение (12), для правой части которого после элементарных преобразований получаем следующую границу сверху:

$$
\left(\frac{V_{n}}{V_{n}+U_{n}}\right)^{u-1} \sum_{k=0}^{m-1}\left(\begin{array}{l}
m \\
k
\end{array}\right) \frac{m-k}{\left(V_{n}+U_{n}\right)^{m-k}} \max _{i \leqslant m-k-1}\left(\left(\begin{array}{c}
u-1 \\
i
\end{array}\right)\left(\frac{U_{n}}{V_{n}}\right)^{i}\right)
$$

Учитывая неравенство

$$
\left(\begin{array}{c}
u-1 \\
i
\end{array}\right)\left(U_{n} V_{n}^{-1}\right)^{i}<\frac{\left(\exp (\theta n) m U_{n} V_{n}^{-1}\right)^{i}}{i !}
$$

и то, что $\left(\begin{array}{c}u-1 \\ i\end{array}\right)\left(U_{n} V_{n}^{-1}\right)^{i}$ возрастает по $i$ при $0 \leqslant i \leqslant m$, а также используя оценку

$$
i !>C_{1} e^{-i} i^{i}
$$

справедливую при некотором $C_{1}>0$ и всех $i>0$, и неравенство

$$
\left(\begin{array}{l}
i \\
j
\end{array}\right) \leqslant 2^{i}
$$

запишем для верхней границы (19) следующую оценку сверху:

$$
\begin{aligned}
& \frac{1}{V_{n}+U_{n}}\left(\frac{V_{n}}{V_{n}+U_{n}}\right)^{u-1} \frac{m^{2} 2^{m}}{m !}\left(\frac{e^{\theta n} m U_{n}}{V_{n}}\right)^{m} \\
& \quad \leqslant \frac{C_{1}}{V_{n}+U_{n}}\left(1-\frac{U_{n}}{V_{n}}\right)^{e^{\theta n} m / 2-1}\left(\frac{V_{n}}{V_{n}+U_{n}}\right)^{e^{\theta n} m / 2} m^{2} 2^{m}\left(\frac{e^{\theta n+1} U_{n}}{V_{n}}\right)^{m} .
\end{aligned}
$$

Ввиду неравенства

$$
\ln (1-x) \leqslant-x
$$

справедливого при $x>0$, получаем, что

$$
\ln \left(1-\frac{U_{n}}{V_{n}}\right)\left(\frac{e^{\theta n} m}{2}-1\right)=-(1+o(1)) \frac{U_{n} e^{\theta n} m}{2 V_{n}}
$$


откуда получаем для выражения (20) следующую асимптотическую оценку сверху:

$$
\frac{C_{1}(2 e)^{m} m^{2}}{V_{n}+U_{n}} \exp \left(-m f\left(\frac{U_{n} e^{\theta n}}{V_{n}}\right)\right)\left(\frac{V_{n}}{V_{n}+U_{n}}\right)^{e^{\theta n} m / 2},
$$

где

$$
f(x)=\frac{x}{2}-\ln x
$$

Функция $f(x)$ при $x>2$ монотонно возрастает и стремится к бесконечности, следовательно,

$$
f\left(\frac{U_{n} e^{\theta n}}{V_{n}}\right)>f\left(M_{1}\right)
$$

Выбирая $M_{1}$ настолько большим, что

$$
C_{1} e^{-m f\left(M_{1}\right)} m^{2}(2 e)^{m}<1
$$

при всех $m$, получаем для выражения (21) следующую верхнюю оценку:

$$
\frac{1}{V_{n}+U_{n}}\left(\frac{V_{n}}{V_{n}+U_{n}}\right)^{e^{\theta n}}=\mathbf{P}\left(Z_{n}^{(1)} \geqslant e^{\theta n}\right) .
$$

Математическое ожидание $\mathbf{E}\left(\mathbf{P}\left(Z_{n}^{(1)} \geqslant e^{\theta n} \mid \eta\right) I_{S_{n}<\theta n-M}\right)$, как было отмечено в начале раздела 3 , может быть сделано сколь угодно малым за счет выбора $M$. Выберем $M_{1}, M$ таким образом, чтобы выполнялось неравенство

$$
\mathbf{E}\left(\mathbf{P}\left(Z_{n}^{(1)}+\ldots+Z_{n}^{(m)} \geqslant e^{\theta n} m \mid \eta\right) I_{S_{n}<\theta n-M} I_{U_{n} / V_{n} \geqslant M_{1} e^{-\theta n}}\right)<\frac{\varepsilon}{10} \mathbf{P}\left(S_{n} \geqslant \theta n\right),
$$

что и дает нужную оценку для первой части математического ожидания (18).

Следующие леммы 2, 3 позволяют оценить вторую часть математического ожидания (18) и тем самым закончить доказательство леммы 1. Их доказательство будет проведено для дробно-линейного случая.

Лемма 2. В условиях теоремь 2 найдется такое $N$, что при всех $n>N$ для любых $\varepsilon>0$ и $M_{1}>0$ найдется такое $M$, что при всех $\theta$ из (6) справедливо неравенство

$$
\mathbf{P}\left(\frac{U_{n}}{V_{n}}<M_{1} e^{-\theta n}, S_{n} \leqslant \theta n-M\right)<\frac{\varepsilon}{10} \mathbf{P}\left(S_{n} \geqslant \theta n\right) .
$$

Доказательство. Запишем представление

$$
V_{n} U_{n}^{-1}=\sum_{i=0}^{n-1} e^{S_{n}-S_{i}+\zeta_{i}} .
$$

Таким образом, пара $\left(V_{n} U_{n}^{-1}, S_{n}\right)$ распределена так же, как пара $\left(\sum_{i=1}^{n} e^{\zeta_{n-i}+\widetilde{S}_{i}}, S_{n}\right)$, где $\widetilde{S}_{i}=X_{n}+\ldots+X_{n-i+1}$. Поэтому рассматриваемая в лемме 2 вероятность равна $\mathbf{P}\left(\sum_{i=1}^{n} e^{\tilde{S}_{i}+\zeta_{n-i}}>M_{1}^{-1} e^{\theta n}, S_{n} \leqslant \theta n-M\right)$.

Для удобства везде ниже будем писать вместо $\widetilde{S}_{i}$ писать $S_{i}$. Оценим эту вероятность с помощью следующей леммы. 
Лемма 3. Для любого $\varepsilon>0$ найдутся такие $M_{2}, \delta<\theta$, что справедливо неравенство

$$
\mathbf{P}\left(\exists i \leqslant n: S_{i}+\zeta_{n-i}>\delta n+(\theta-\delta) i+M_{2}\right) \leqslant \varepsilon \mathbf{P}\left(S_{n} \geqslant \theta n\right) .
$$

Доказательство. Фиксируем некоторое $0<\varkappa<1$ и воспользуемся следующими оценками:

$$
\begin{array}{r}
\mathbf{P}\left(\exists i \leqslant n(1-\varkappa): S_{i}+\zeta_{n-i}>\delta n+(\theta-\delta) i+M_{2}\right) \\
\leqslant \mathbf{P}\left(\exists i \leqslant n(1-\varkappa): S_{i}>(\delta-\varkappa(\theta-\delta) / 2) n+(\theta-\delta+\varkappa(\theta-\delta) / 2) i+M_{2}\right) \\
\quad+\mathbf{P}\left(\max _{i \leqslant n(1-\varkappa)} \zeta_{n-i}>\varkappa(1-\varkappa)(\theta-\delta) n / 2\right) .
\end{array}
$$

Полагая для краткости

$$
\tilde{\delta}=(\delta-\varkappa(\theta-\delta) / 2)
$$

оценим сверху вероятностью $\mathbf{P}\left(\max _{i \leqslant n(1-\varkappa)} \sum_{j=1}^{i}\left(X_{j}-\theta+\delta\right) \geqslant \tilde{\delta} n+M_{2}\right)$ первую вероятность в правой части (23). Величины

$$
\tilde{X}_{j}=X_{j}-\theta+\delta
$$

имеют преобразование Лапласа

$$
\widetilde{R}(h)=R(h) e^{-h(\theta-\delta)},
$$

так что

$$
\widetilde{R}\left(\tilde{h}_{\delta}\right)=e^{-h_{\theta}(\theta-\delta)} R\left(h_{\theta}\right), \quad \tilde{\Lambda}(\delta)=\Lambda(\theta),
$$

где $\tilde{h}_{\delta}, \tilde{\Lambda}(\delta)$ по отношению к случайным величинам $\tilde{X}_{j}$ имеют тот же смысл, что и $h_{\theta}$, $\Lambda(\theta)$ для случайных величин $X_{j}$. Выбирая $\delta$ достаточно малым, мы видим, что $\widetilde{R}\left(\tilde{h}_{\delta}\right)>1$ при всех $\theta \in\left[\theta_{1}, \theta_{2}\right]$; так что, применяя (5), приходим к соотношениям

$$
\mathbf{P}\left(\exists i: S_{i}>\delta n+(\theta-\delta) i+M_{2}\right)=(1+o(1)) C(\theta, \delta) n^{-1 / 2} e^{-\Lambda(\theta) n} e^{-h_{\theta} M_{2}} .
$$

Полагая для краткости

$$
\tilde{\theta}=\varkappa(1-\varkappa)(\theta-\delta) / 2
$$

оценим вторую вероятность в правой части (23):

$$
\mathbf{P}\left(\max _{i \leqslant n(1-\varkappa)} \zeta_{n-i}>\tilde{\theta} n\right)=1-\left(1-\mathbf{P}\left(\zeta_{i}>\tilde{\theta} n\right)\right)^{n} \leqslant n \mathbf{P}\left(e^{h \zeta_{i}}>e^{h \tilde{\theta} n}\right) \leqslant n \mathbf{E} e^{h \zeta_{i}} e^{-h \tilde{\theta} n} .
$$

Выбирая $h$ достаточно большим, видим, что

$$
\mathbf{P}\left(\max _{i \leqslant n(1-\varkappa)} \zeta_{n-i}>\varkappa(1-\varkappa)(\theta-\delta) n / 2\right) \leqslant \frac{\varepsilon}{4} \mathbf{P}\left(S_{n} \geqslant \theta n\right) .
$$

Подставляя неравенства (24), (25) в (23), получаем, что

$$
\mathbf{P}\left(\exists i \leqslant n(1-\varkappa): S_{i}+\zeta_{n-i}>\delta n+(\theta-\delta) i+M_{2}\right) \leqslant \frac{\varepsilon}{2} \mathbf{P}\left(S_{n} \geqslant \theta n\right) .
$$


Покажем, что параметры $\delta$ и $\varkappa$ могут быть выбраны так, чтобы выполнялось неравенство

$$
\mathbf{P}\left(\exists i: n(1-\varkappa) \leqslant i \leqslant n: S_{i}+\zeta_{n-i}>\delta n+(\theta-\delta) i+M_{2}\right) \leqslant \frac{\varepsilon}{2} \mathbf{P}\left(S_{n} \geqslant \theta n\right),
$$

и тем самым закончим доказательство леммы 3.

Вероятность в правой части (27) оценивается сверху суммой

$$
\sum_{i=n(1-\varkappa)}^{n} \int_{\mathbf{R}} \mathbf{P}\left(S_{i-1}>\delta n+(\theta-\delta) i+M_{2}-\ln a(x)-\ln \frac{b(x)}{2 a^{2}(x)}\right) \mathbf{P}\left(\eta_{n-i} \in d x\right) .
$$

В силу неравенства (25), при достаточно больших $n$ справедлива оценка

$$
\mathbf{P}\left(\max _{i \leqslant n} \zeta_{i}>\theta \varkappa n\right)<\frac{\varepsilon}{4} \mathbf{P}\left(S_{n} \geqslant \theta n\right),
$$

а в силу неравенства Чебышева

$$
\mathbf{P}\left(\exists i<n: X_{i}>\theta n\right) \leqslant n \mathbf{E} \exp \left(h_{\theta} X-h_{\theta} \theta n\right)=\varepsilon \mathbf{P}\left(S_{n} \geqslant \theta n\right) .
$$

В итоге, сумма (28) оценивается сверху величиной

$$
\begin{aligned}
\frac{\varepsilon}{4} \mathbf{P}\left(S_{n} \geqslant \theta n\right)+\sum_{i=n(1-\varkappa)}^{n} \int_{\mathbf{R}} \mathbf{P}\left(\eta_{n-i}\right. & \in d x) I_{\ln \left(b(x)(2 a(x))^{-1}\right)<\theta n(1-2 \varkappa)} \\
& \times \mathbf{P}\left(S_{i-1}>\delta n+(\theta-\delta) i+M_{2}-\ln \frac{b(x)}{2 a(x)}\right) .
\end{aligned}
$$

Полагая

$$
\tilde{\theta}_{2}=\delta n /(i-1)+\left((\theta-\delta) i-\ln \left(b(x)(2 a(x))^{-1}\right)\right) /(i-1),
$$

замечаем, что для таких $x$, что $\ln \left(b(x)(2 a(x))^{-1}\right)<\theta n(1-2 \varkappa), \theta \in\left[\theta_{1}, \theta_{2}\right], \tilde{\theta}_{2}$ содержится в некотором отрезке $\left[\theta_{3}, \theta_{4}\right] \subset\left(0, m^{+}\right)$, не зависящем от $x$. Представляя вероятность в правой части (29) в виде $\mathbf{P}\left(S_{i}>\tilde{\theta}_{2} i+M_{2}\right)$ и применяя к ней теорему Петрова (4), получаем, что сумма в правой части (12) асимптотически оценивается сверху величиной

$$
e^{-h_{\theta} M_{2}} \sum_{i=n(1-\varkappa)}^{n} i^{-1 / 2} \int_{\mathbf{R}} \mathbf{P}\left(\eta_{n-i} \in d x\right) e^{-\Lambda\left(\tilde{\theta}_{2}\right)(i-1)} C\left(\tilde{\theta}_{2}\right) .
$$

Используя неравенство Гёльдера и неравенство

$$
\Lambda\left(\tilde{\theta}_{2}\right)(i-1)>\Lambda(\theta) i+h_{\theta} \delta(n-i)-h_{\theta} \ln \frac{b(x)}{2 a^{2}(x)},
$$

вытекающее из выпуклости функции $\Lambda(x)$, и выбирая $\delta<\theta$ достаточно близким к $\theta$ так, чтобы выполнялось неравенство

$$
h_{\theta} \delta>\Lambda(\theta)=h_{\theta} \theta-\ln R\left(h_{\theta}\right),
$$

получаем, что (30) оценивается сверху величиной

$$
n^{-1 / 2} e^{-\Lambda(\theta) n} e^{-h_{\theta} M_{2}} C_{1}\left(\mathbf{E}\left(e^{h_{\theta} p \zeta_{1}}\right)\right)^{1 / p}\left(\mathbf{E}\left(e^{h_{\theta} q X_{1}}\right)\right)^{1 / q} \sum_{i=0}^{\infty} e^{-\left(h_{\theta} \delta-\Lambda(\theta)\right) i},
$$


где

$$
\frac{1}{p}+\frac{1}{q}=1, \quad p, q>1
$$

а $q$ выбирается из условия

$$
\frac{h_{\theta_{2}}}{q}<h^{+}
$$

$C_{1}$ - некоторая величина, не зависящая от $n, M_{2}$ и $\theta$. Выбирая $M_{2}$ в (31) достаточно большим, приходим к неравенству (10). Лемма 3 доказана.

В силу леммы 3 , при подходящих значениях параметров $M_{2}, \delta$ справедливо неравенство

$$
\begin{aligned}
& \mathbf{P}\left(\sum_{i=0}^{n-1} e^{S_{i}+\zeta_{n-i}>}\right.\left.M_{1}^{-1} e^{\theta n}, S_{n} \leqslant \theta n-M\right) \leqslant \frac{\varepsilon}{30} \mathbf{P}\left(S_{n} \geqslant \theta n\right) \\
&+\mathbf{P}\left(\sum_{i=0}^{n-1} e^{S_{i}+\zeta_{n-i}>M_{1}^{-1} e^{\theta n}, S_{n} \leqslant \theta n-M},\right. \\
&\left.S_{i}+\zeta_{n-i} \leqslant \delta n+(\theta-\delta) i+M_{2} \text { для всех } i \leqslant n\right) .
\end{aligned}
$$

В предположении, что при всех $i \leqslant n$ выполнено неравенство

$$
S_{i}+\zeta_{n-i} \leqslant \delta n+(\theta-\delta) i+M_{2}
$$

справедлива следующая оценка:

$$
\sum_{i=0}^{n-l} e^{S_{i}+\zeta_{n-i}} \leqslant \sum_{i=0}^{n-l} e^{\delta n+(\theta-\delta) i+M_{2}} \leqslant e^{M_{2}} e^{\theta n} e^{-(\theta-\delta) l}\left(1-e^{-(\theta-\delta)}\right)^{-1}
$$

Выбирая $l$ достаточно большим, заключаем, что

$$
\sum_{i=0}^{n-l} e^{S_{i}+\zeta_{n-i}} \leqslant \frac{1}{2} e^{\theta n} M_{1}^{-1}
$$

Отсюда и из (32) следует, что левая часть (15) оценивается сверху величиной

$$
\begin{aligned}
\frac{\varepsilon}{30} \mathbf{P}\left(S_{n} \geqslant \theta n\right)+ & \mathbf{P}\left(\sum_{i=n-l}^{n-1} e^{S_{i}+\zeta_{n-i}}>\frac{M_{1}^{-1} e^{\theta n}}{2},\right. \\
& +\mathbf{P a x}\left(S_{n-l+1}, \ldots, S_{n}\right)+\max \left(S_{n-l+1}, \ldots, S_{n}\right)+\max \left(\zeta_{n-l+1}, \ldots, \zeta_{n}\right)>\theta n-M_{2}, \\
& \left.\quad M_{n} \leqslant \theta n-M_{3}\right) \\
& +\mathbf{P}\left(S_{n} \leqslant \theta n-M, M_{n}>\theta n-M_{3}\right),
\end{aligned}
$$

где $M_{2}-$ некоторая константа. 
Второе слагаемое суммы (33) при $M_{2}>\ln M_{1}+\ln l$ равняется нулю в силу неравенств

$$
\begin{aligned}
\sum_{i=n-l}^{n-1} e^{S_{i}+\zeta_{n-i}} & \leqslant l e^{\max \left(S_{n-l+1}, \ldots, S_{n}\right)+\max \left(\zeta_{n-l+1}, \ldots, \zeta_{n}\right)} \\
& \leqslant l e^{\theta n} e^{-M_{2}}<\frac{M_{1}^{-1} e^{\theta n}}{2} .
\end{aligned}
$$

Если положить

$$
\begin{aligned}
& f\left(x_{1}, \ldots, x_{l}\right)=\sum_{i=1}^{l} \ln a\left(x_{i}\right), \\
& g\left(x_{1}, \ldots, x_{l}\right)=\max _{i \leqslant l} \frac{b\left(x_{i}\right)}{a^{2}\left(x_{i}\right)},
\end{aligned}
$$

то третье слагаемое в (33) может быть оценено величиной

$$
\begin{aligned}
& \mathbf{P}\left(\max \left(S_{n-l+1}, \ldots, S_{n}\right)+\max \left(\zeta_{n-l+1}, \ldots, \zeta_{n}\right)>\theta n-M_{2}, M_{n} \leqslant \theta n-M_{3}\right) \\
& \leqslant \int_{\mathbf{R}}\left(\mathbf{P}\left(M_{n-l} \geqslant \theta n-f\left(x_{1}, \ldots, x_{l}\right)-g\left(x_{1}, \ldots, x_{l}\right)-M_{2}\right)\right. \\
& \left.\quad-\mathbf{P}\left(M_{n-l}>\theta n-M_{3}-\sum_{i=1}^{l} a\left(x_{i}\right)\right)\right) I_{g\left(x_{1}, \ldots, x_{l}\right)>M_{3}-M_{2}} \mathbf{P}\left(\eta_{i} \in d x_{i}, i \leqslant l\right) .
\end{aligned}
$$

Аналогично тому, как это было сделано в (29), добавим под знак интеграла в (34) индикатор события $\left\{f\left(x_{1}, \ldots, x_{l}\right)+g\left(x_{1}, \ldots, x_{l}\right) \leqslant(\theta-x) n\right\}$. Подставляя в (34) соотношение (5) и пользуясь тем, что соответствующие асимптотические оценки равномерны по $\theta \in\left[\theta_{1}, \theta_{2}\right]$, находим, что правая часть (34) асимптотически оценивается сверху величиной

$$
\begin{aligned}
& D(\theta) n^{-1 / 2} \int_{\mathbf{R}} e^{-\Lambda\left(\theta-f\left(x_{1}, \ldots, x_{l}\right) / n-M_{3} / n\right) n}\left(e^{h_{\theta}\left(M_{2}-M_{3}+g\left(x_{1}, \ldots, x_{l}\right)\right)}-1\right)
\end{aligned}
$$

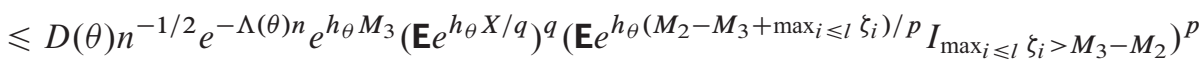

$$
\begin{aligned}
& \leqslant \mathbf{P}\left(M_{n} \geqslant \theta n\right) e^{h_{\theta} M_{2}} \int_{x>M_{3}-M_{2}} e^{h_{\theta} x / p} l \mathbf{P}\left(\zeta_{1} \leqslant x\right)^{l-1} \mathbf{P}\left(\zeta_{1} \in d x\right),
\end{aligned}
$$

где $q>1$ выбирается достаточно близким к 1 так, чтобы выполнялось неравенство

$$
\frac{h_{\theta_{2}}}{q}<h^{+} \text {. }
$$

Интеграл в правой части (35) сходится равномерно по $\theta \in\left[\theta_{1}, \theta_{2}\right]$ и за счет выбора $M_{3}$ может быть сделан сколь угодно малым, а значит, третье слагаемое суммы (33) может быть сделано меньше $(\varepsilon / 30) \mathbf{P}\left(S_{n} \geqslant \theta n\right)$. В силу теоремы 5 из [5], вероятность $\mathbf{P}\left(S_{n} \leqslant \theta n-M, M_{n} \geqslant \theta n-M_{3}\right)$ при любом $M_{3}$ может быть сделана меньше, чем $(\varepsilon / 30) \mathbf{P}\left(S_{n} \geqslant \theta n\right)$ за счет выбора $M$. Подставляя полученные оценки в (33), завершаем доказательство леммы 2.

Следующие леммы 4 и 5 позволяют оценить оставшиеся слагаемые в (17). 
Лемма 4. При $n, m \rightarrow \infty$ справедливы следующие соотношения:

$$
\begin{aligned}
& \mathbf{E}\left(\mathbf{P}\left(Z_{n}^{(1)}+\ldots+Z_{n}^{(m)} \geqslant e^{\theta n} m \mid \eta\right) I_{\theta n-M \leqslant S_{n}<\theta n-\tilde{\varepsilon}}\right)=\varepsilon \mathbf{P}\left(S_{n} \geqslant \theta n\right), \\
& \mathbf{E}\left(\mathbf{P}\left(Z_{n}^{(1)}+\ldots+Z_{n}^{(m)} \geqslant e^{\theta n} m \mid \eta\right) I_{\theta n+\tilde{\varepsilon} \leqslant S_{n}<\theta n+M}\right) \sim \mathbf{P}\left(\tilde{\varepsilon} \leqslant S_{n}-\theta n \leqslant M\right) .
\end{aligned}
$$

Доказательство. Полагая

$$
\begin{aligned}
\widetilde{Z}_{n}^{(i)} & =\frac{Z_{n}^{(i)}-e^{S_{n}}}{e^{S_{n}} \sqrt{2 V_{n}-1+e^{-S_{n}}}}, \\
A_{n} & =\left\{\theta n-M \leqslant S_{n}<\theta n-\tilde{\varepsilon}, \min _{i \leqslant n} S_{i}>-\ln m / 4\right\},
\end{aligned}
$$

мы видим, что левая часть (36) оценивается сверху величиной

$$
\begin{array}{r}
\mathbf{E}\left(\mathbf{P}\left(\tilde{Z}_{n}^{(1)}+\ldots+\widetilde{Z}_{n}^{(m)} \geqslant \frac{\left(e^{\theta n-S_{n}}-1\right) m}{\sqrt{2 V_{n}-1+e^{-S_{n}}}} \mid \eta\right) I_{A_{n}}\right) \\
\quad+\mathbf{P}\left(S_{n} \geqslant \theta n-M, \min _{i \leqslant n} S_{i}<-\ln m / 4\right) .
\end{array}
$$

Нетрудно проверить, что

$$
\begin{aligned}
\mathbf{E}\left(\widetilde{Z}_{n}^{(i)} \mid \eta\right) & =0, \\
\mathbf{E}\left(\left(\widetilde{Z}_{n}^{(i)}\right)^{2} \mid \eta\right) & =1, \\
\mathbf{E}\left(\left|\widetilde{Z}_{n}^{(i)}\right|^{3} \mid \eta\right) & \leqslant \frac{6 V_{n}^{2}+6 V_{n}+4+\left(6 V_{n}+3\right) e^{-S_{n}}+e^{-2 S_{n}}}{\left(2 V_{n}-1+e^{-S_{n}}\right)^{3 / 2}} \\
& \leqslant 6 V_{n}+10+9 e^{-S_{n}}+e^{-2 S_{n}}=D_{n} .
\end{aligned}
$$

Используя неравенство Берри-Эссеена, запишем, что

$$
\left|\mathbf{P}\left(\widetilde{Z}_{n}^{(1)}+\ldots+\widetilde{Z}_{n}^{(m)} \geqslant x \mid \eta\right)-1+\Phi(x)\right| \leqslant \frac{C D_{n}}{\sqrt{m}},
$$

где $C-$ некоторая константа, не зависящая от $\eta$. Таким образом,

$$
\mathbf{E}\left(\mathbf{P}\left(\widetilde{Z}_{n}^{(1)}+\ldots+\widetilde{Z}_{n}^{(m)} \geqslant x \mid \eta\right) I_{A_{n}}\right)=(1-\Phi(x)) \mathbf{P}\left(A_{n}\right)+Q_{n},
$$

где

$$
\left|Q_{n}\right| \leqslant C \mathbf{E}\left(D_{n} I_{A_{n}}\right) m^{-1 / 2}
$$

Заметим, что справедливы неравенства

$$
\begin{gathered}
\mathbf{E}\left(D_{n} I_{A_{n}}\right) \leqslant 20+6 \mathbf{E}\left(V_{n} I_{A_{n}}\right)=20+6 \sum_{i \leqslant n} \mathbf{E}\left(e^{-S_{i}} I_{A_{n}}\right), \\
\mathbf{E}\left(e^{-S_{i}} I_{A_{n}}\right) \leqslant e^{\ln m / 4} \mathbf{P}\left(S_{n} \geqslant \theta n-M, S_{i} \leqslant \tilde{\theta} i\right)+e^{-\tilde{\theta} i / 2} \mathbf{P}\left(S_{n} \geqslant \theta n-M\right),
\end{gathered}
$$

где $\tilde{\theta}$ - некоторая положительная константа. Согласно оценкам, полученным в лемме 1 из [6], $\tilde{\theta}$ может быть выбрано таким образом, что сумма $\sum_{i=1}^{n} \mathbf{P}\left(S_{n} \geqslant \theta n-M, S_{i} \leqslant \tilde{\theta} i\right)$ равномерно ограничена по $n$. Отсюда и из (41) получаем, что

$$
\left|Q_{n}\right|=C m^{-1 / 4} \mathbf{P}\left(S_{n} \geqslant \theta n-M\right), \quad m, n \rightarrow \infty .
$$


Заметим, что при условии события $A_{n}$ справедливы неравенства

$$
\frac{\left(e^{\theta n-S_{n}}-1\right) m}{\sqrt{2 V_{n}-1+e^{-S_{n}}}} \geqslant \frac{\left(e^{\tilde{\varepsilon}}-1\right) m}{2 V_{n}} \geqslant \frac{\tilde{\varepsilon} m}{2 V_{n}} .
$$

Подставляя (42) и (40) в (38), получаем, что

$$
\begin{aligned}
\mathbf{E}\left(\mathbf{P}\left(Z_{n}^{(1)}+\ldots+Z_{n}^{(m)} \geqslant e^{\theta n} m \mid \eta\right) I_{A_{n}}\right) \leqslant(1 & -\Phi(\sqrt{m} \tilde{\varepsilon})) \mathbf{P}\left(S_{n} \geqslant \theta n-M\right) \\
& +C m^{-1 / 4} \mathbf{P}\left(S_{n} \geqslant \theta n-M\right) \\
& +\mathbf{P}\left(2 V_{n} \geqslant \sqrt{m}, S_{n} \geqslant \theta n-M\right) \\
& +\mathbf{P}\left(M_{n} \geqslant \theta n-M+\ln m / 4\right) \\
= & \varepsilon \mathbf{P}\left(S_{n} \geqslant \theta n\right)
\end{aligned}
$$

при $m, n \rightarrow \infty$, и соотношение (36) доказано. Соотношение (37) доказывается аналогично. Полагая

$$
B_{n}=\left\{\theta n+\tilde{\varepsilon} \leqslant S_{n}<\theta n+M, \min _{i \leqslant n} S_{i} \geqslant-\ln m / 4\right\},
$$

получаем, что при условии $B_{n}$

$$
\frac{\left(e^{\theta n-S_{n}}-1\right) m}{\sqrt{2 V_{n}-1+e^{-S_{n}}}} \leqslant-\frac{\tilde{\varepsilon} m}{2 V_{n}},
$$

и, подобно тому, как мы из (42) выводили (43), из (44) получаем, что

$$
\begin{aligned}
\mathbf{E}\left(\mathbf{P}\left(Z_{n}^{(1)}+\ldots+Z_{n}^{(m)} \geqslant e^{\theta n} m \mid \eta\right) I_{B_{n}}\right) \geqslant 1 & -\Phi(-\sqrt{m} \tilde{\varepsilon}) \mathbf{P}\left(\tilde{\varepsilon} \leqslant S_{n}-\theta n<M\right) \\
& -c_{m} \mathbf{P}\left(S_{n} \geqslant \theta n-M\right) \\
& -\mathbf{P}\left(2 V_{n} \geqslant \sqrt{m}, S_{n} \geqslant \theta n-M\right) \\
= & 1-\varepsilon \mathbf{P}\left(S_{n} \geqslant \theta n\right),
\end{aligned}
$$

где $c_{n}=O\left(n^{-1 / 2}\right)$.

Следующая лемма 5 получается непосредственным применением теоремы Петрова.

Лемма 5. Найдутся такие $\tilde{\varepsilon}, M, N_{1}, M_{1}$, что для всех $n>N_{1}$ и для всех $m>M_{1}$ справедливы оценки

$$
\begin{array}{r}
\mathbf{E}\left(\mathbf{P}\left(Z_{n}^{(1)}+\ldots+Z_{n}^{(m)} \geqslant e^{\theta n} m \mid \eta\right) I_{\theta n-\tilde{\varepsilon} \leqslant S_{n} \leqslant \theta n+\tilde{\varepsilon}}\right) \leqslant \varepsilon \mathbf{P}\left(S_{n} \geqslant \theta n\right) / 5, \\
\mathbf{E}\left(\mathbf{P}\left(Z_{n}^{(1)}+\ldots+Z_{n}^{(m)} \geqslant e^{\theta n} m \mid \eta\right) I_{S_{n}} \geqslant \theta n+M\right)
\end{array}
$$

Пользуясь леммами $1-5$, из (17) выводим, что для произвольного $\varepsilon$ найдутся такие $M_{1}, N_{1}, M, \tilde{\varepsilon}$, что для всех $m>M_{1}$ и для всех $n>N_{1}$ справедлива оценка

$$
\left|\mathbf{P}\left(Z_{n} \geqslant m e^{\theta n} \mid Z_{0}=m\right)-\mathbf{P}\left(\tilde{\varepsilon} \leqslant S_{n}<M\right)\right| \leqslant \varepsilon \mathbf{P}\left(S_{n} \geqslant \theta n\right) .
$$

Осуществляя в (45) предельный переход при $\varepsilon \rightarrow 0, M \rightarrow \infty, \tilde{\varepsilon} \rightarrow 0$, приходим к теореме 2 .

Пользуясь случаем, автор выражает признательность М. В. Козлову за обсуждение работы, а также благодарит рецензента за крайне внимательное прочтение работы и ряд ценных замечаний и исправлений. 


\section{Список литературы}

1. Козлов М. В., О больших уклонениях ветвящихся процессов. Дискретная математика (2006) 18, №2, 29-47.

2. Козлов М. В., О больших уклонениях строго докритических ветвящихся процессов в случайной среде с геометрическим распределением числа потомков. Теория вероятностей и ее применения (2009) 54, №3, 439-465.

3. Agresti A., On the extinction times of varying and random enviroment branching processes. J. Appl. Probab. (1975) 12, 39-46.

4. Петров В. В., О вероятностях больших уклонений сумм независимых случайных величин. Теория вероятностей и ее применения (1965) 10, 310-322.

5. Шкляев А. В., Предельные теоремы для случайного блуждания при условии большого уклонения максимума. Теория вероятностей и ее применения (2010) 55, №3, 590-598.

6. Шкляев А. В., Большие уклонения статистики Шеппа. Теория вероятностей и ее применения (2010) 55, №4, 796-803.

7. Boinghoff C., Kersting G., Upper large deviations of branching processes in a random environmentoffspring distributions with geometrically bounded tails. Stochastic Process. Appl. (2010) 10, 2064 2077.

8. Bansaye V., Berestycki J., Large deviations for branching processes in random environment. Markov Process. Relat. Fields (2009) 4, 493-524.

Статья поступила 20.12.2011. Переработанный вариант поступил 24.07.2012. 